\title{
EAN/ERS/ESO/ESRS statement on the impact of sleep disorders on risk and outcome of stroke
}

\author{
Claudio L.A. Bassetti ${ }^{1,2,23}$, Winfried Randerath ${ }^{3,23}$, Luca Vignatelli $\mathbb{1}^{4}$, \\ Luigi Ferini-Strambi ${ }^{5}$, Anne-Kathrin Brill ${ }^{6}$, Maria R. Bonsignore (1) ${ }^{7}$, \\ Ludger Grote ${ }^{8}$, Poul Jennum ${ }^{9}$, Didier Leys ${ }^{10}$, Jens Minnerup ${ }^{11}$, Lino Nobili ${ }^{12}$, \\ Thomy Tonia ${ }^{13}$, Rebecca Morgan ${ }^{14}$, Joel Kerry ${ }^{15}$, Renata Riha ${ }^{16,17}$, \\ Walter T. McNicholas (18) 18,20,24 and Vasileios Papavasileiou ${ }^{21,22,24}$
}

@ERSpublications

Evidence suggests a bidirectional relationship between sleep and stroke. However, the pathophysiological base of the associations and the possibilities of improving prevention and outcome through sleep-related interventions require further evaluation. http://bit.ly/36De7Cy

Cite this article as: Bassetti CLA, Randerath W, Vignatelli L, et al. EAN/ERS/ESO/ESRS statement on the impact of sleep disorders on risk and outcome of stroke. Eur Respir J 2020; 55: 1901104 [https://doi.org/ 10.1183/13993003.01104-2019].

ABSTRACT Sleep disorders are highly prevalent in the general population and may be linked in a bidirectional fashion to stroke, which is one of the leading causes of morbidity and mortality.

Four major scientific societies established a task force of experts in neurology, stroke, respiratory medicine, sleep medicine and methodology, to critically evaluate the evidence regarding potential links and the impact of therapy. 13 research questions were evaluated in a systematic literature search using a stepwise hierarchical approach: first, systematic reviews and meta-analyses; second, primary studies postdating the systematic reviews/meta-analyses. A total of 445 studies were evaluated and 88 included. Statements were generated regarding current evidence and clinical practice.

Severe obstructive sleep apnoea (OSA) doubles the risk for incident stroke, especially in young to middle-aged patients. Continuous positive airway pressure (CPAP) may reduce stroke risk, especially in treatment-compliant patients. The prevalence of OSA is high in stroke patients and can be assessed by polygraphy. Severe OSA is a risk factor for recurrence of stroke and may be associated with stroke mortality, while CPAP may improve stroke outcome. It is not clear if insomnia increases stroke risk, while pharmacotherapy of insomnia may increase it. Periodic limb movements in sleep (PLMS), but not restless limb syndrome (RLS), may be associated with an increased risk of stroke. Preliminary data suggest a high frequency of post-stroke insomnia and RLS and their association with a less favourable stroke outcome, while treatment data are scarce.

Overall, the evidence base is best for OSA relationship with stroke and supports active diagnosis and therapy. Research gaps remain especially regarding insomnia and RLS/PLMS relationships with stroke.

This article has an editorial commentary: https://doi.org/10.1183/13993003.01977-2019

This document was endorsed by the ERS Executive Committee on 17 October 2019, and by the EAN on 20 May 2019, the ESO on 4 June 2019, and ESRS on 22 May 2019.

This article has supplementary material available from erj.ersjournals.com

Received: 5 June 2019 | Accepted after revision: 12 Sept 2019

The article has been co-published with permission in the European Respiratory Journal and the European Journal of Neurology. The articles are identical except for minor stylistic and spelling differences in keeping with each journal's style. Either citation can be used when citing this article. Copyright @European Academy of Neurology and European Respiratory Society 2020. 


\section{Introduction}

Stroke is defined as an acute and focal neurological brain damage due to ischaemia or haemorrhage. It represents the second most common cause of death and the third most common cause of disability-adjusted life-years worldwide [1]. The burden of stroke is greater in younger people and the incidence of stroke is increasing in this group [1]. Stroke is a heterogeneous condition with a variety of underlying mechanisms and causes. The risk of stroke is affected by non-modifiable (e.g. age, family history) and potentially modifiable factors (e.g. hypertension, current smoking, alcohol consumption, obesity, diabetes, lack of physical activity, psychosocial stress and depression) [2].

Sleep-wake disorders (SWD) are very frequent in the general population and have a major impact on performance, quality of life as well as overall health status. The most common SWD are sleep disordered breathing (SDB), insomnia and sleep-related movement disorders, such as restless limb syndrome (RLS) and periodic limb movements in sleep (PLMS) [3].

SDB is highly prevalent and may present as obstructive or central apnoeas and hypopnoeas, and/or hypoventilation. The obstructive form is most common and recent population-based epidemiological studies have reported a prevalence of obstructive apnoeas and hypopnoeas per hour of sleep (AHI) $\geqslant 15$ in up to $25 \%$ of women and $50 \%$ of men. The prevalence increases with age $[4,5]$. Benjafield et al. [6] recently described a global prevalence of 425 million (399-450) adults aged 30-69 years having an AHI $>15$ events per $h$.

The association of obstructive SDB and clinical symptoms, defined as obstructive sleep apnoea syndrome (OSA), is less prevalent, affecting about $10 \%$ of the male and $5 \%$ of the female population $[4,7]$. Associations are reported between OSA and a wide range of behavioural and clinical disorders that include fatigue, excessive daytime sleepiness (EDS), insomnia and non-restorative sleep, attention and cognitive deficits, increased frequency of accidents, in addition to cardiovascular disorders, hypertension, diabetes mellitus and the metabolic syndrome, and depression $[4,8,9]$. Cluster analysis of large databases have demonstrated different clinical phenotypes of OSA according to symptoms (sleepiness, insomnia, depression), anthropometric factors (age, gender, body constitution) and comorbidities [10-12].

Insomnia is defined by complaints of difficulties initiating sleep, maintaining sleep or waking up too early, and their association with diurnal symptoms such as fatigue, sleepiness, attention/concentration problems, impaired social or occupational performance, and mood disturbance [13]. In this context it is important to stress the fact that 1) the current definition of insomnia does not require an objective shortening of sleep duration and that 2) short sleepers without diurnal complaints are not classified as insomniacs. Insomnia affects over $20 \%$ of the general population and up to $5-10 \%$ use hypnotics [13], with a tendency in recent years to an increase in some countries including the USA and an increase in benzodiazepine-related deaths [14]. Associations have been reported between insomnia and depression, anxiety, hypertension, diabetes and cardiovascular disorders [15-19].

Affiliations: ${ }^{1}$ Neurology Dept, Medical Faculty, University Hospital, Bern, Switzerland. ${ }^{2}$ Dept of Neurology Sechenov First Moscow State Medical University, Moscow, Russia. ${ }^{3}$ Bethanien Hospital, Clinic of Pneumology and Allergology, Center for Sleep Medicine and Respiratory Care, Institute of Pneumology at the University of Cologne, Solingen, Germany. "Servizio di Epidemiologia e Biostatistica IRCCS, Istituto delle Scienze Neurologiche di Bologna Ospedale Bellaria, Bologna, Italy. ${ }^{5}$ Dept of Neurology OSR-Turro, Sleep Disorder Center, Vita-Salute San Raffaele University, Milan, Italy. ${ }^{6}$ Dept of Pulmonary Medicine, University and University Hospital Bern, Bern, Switzerland. ${ }^{7}$ PROMISE Dept, Division of Respiratory Medicine, DiBiMIS, University of Palermo and IBIM-CNR, Palermo, Italy. ${ }^{8}$ Sleep Disorders Center, Dept of Pulmonary Medicine, Sahlgrenska University Hospital, Göteborg, Sweden. ${ }^{9}$ Danish Center for Sleep Medicine, Rigshospitalet, Copenhagen, Denmark. ${ }^{10}$ Dept of Neurology, University of Lille, Lille, France. ${ }^{11}$ Dept of Neurology and Institute for Translational Neurology, University of Muenster, Muenster, Germany. ${ }^{12}$ Child Neuropsychiatry Unit, Gaslini Institute DINOGMI, University of Genova, Genoa, Italy. ${ }^{13}$ Institute of Social and Preventive Medicine, Universtity of Bern, Bern, Switzerland. ${ }^{14}$ Dept of Health Research Methods, Evidence, and Impact, McMaster University, Hamilton, ON, Canada. ${ }^{15}$ Library and Information Service, Leeds Teaching Hospitals NHS Trust, Leeds, UK. ${ }^{16}$ Sleep Research Unit, Centre for Clinical Brain Sciences, University of Edinburgh, Edinburgh, UK. ${ }^{17}$ Dept of Sleep Medicine, Royal Infirmary of Edinburgh, Edinburgh, UK. ${ }^{18}$ Dept of Respiratory and Sleep Medicine, St. Vincent's University Hospital, Dublin, Ireland. ${ }^{19}$ 'School of Medicine, University College Dublin, Dublin, Ireland. ${ }^{20}$ First Affiliated Hospital of Guangzhou Medical University, Guangzhou, China. ${ }^{21}$ Leeds Teaching Hospital NHS Trust, Leeds, UK. ${ }^{22}$ Medical School, University of Leeds, Leeds, UK. ${ }^{23}$ Co-shared first authorship. ${ }^{24} \mathrm{Co}$-shared senior authorship.

Correspondence: Winfried Randerath, Bethanien Hospital, University of Cologne, Pneumology, Aufderhoeher Str. 169, Solingen 42699, Germany. E-mail: randerathlaklinik-bethanien.de

Correspondence: Claudio Bassetti, Neurology Dept, Inselspital, University of Bern, Freiburgstrasse 18, 3010 Bern, Switzerland. E-mail: Claudio.Bassettiainsel.ch 
RLS is defined by the presence of an urge to move the limbs (usually the legs), accompanied by unpleasant sensations, which worsen during rest, in the evening or at night, and are relieved by movement [20, 21]. A positive family history and response to dopaminergic drugs, as well as the presence of periodic limb movements during wakefulness or sleep, support the diagnosis of RLS [21]. The frequency of RLS in the general population is as high as $5-15 \% ; 2-5 \%$ present with frequent or severe symptoms [22]. An association has been reported between RLS and insomnia, EDS, iron deficiency/anaemia, uraemia, hypertension, diabetes, depression, pain, neurological disorders, COPD and cardiovascular disorders [23-25].

Other SWD such as extremes of sleep duration (short and long sleep) as well as circadian rhythm disorders (e.g. related to shift work) have been linked to cardiovascular diseases, such as stroke, and have been suggested to negatively influence their course and outcome [26-35].

Accordingly, the American Heart Association suggested diagnosis and treatment strategies for SWD in the management of stroke [36]. However, the extent and relevance of this bi-directional relationship between sleep and stroke remains a matter of debate.

In order to assess the current knowledge about the link between sleep disorders and stroke, and motivated by previous successful multidisciplinary activities [3,9], the European Academy of Neurology (EAN), the European Respiratory Society (ERS), the European Sleep Research Society (ESRS), and the European Stroke Organization (ESO) created a task force to produce a common statement on this topic.

\section{Methods}

The multidisciplinary task force was composed of 15 experts in neurology, pneumology, sleep medicine, stroke and methodology. It followed the recommendations of the EAN and ERS statement/consensus review documents (for details of the process please refer to the supplementary material) [37-39].

The task force developed a document focusing on the following main topics:

1) Sleep-wake disorders, such as sleep-related breathing disorders, insomnia and restless legs syndrome/ periodic limb movement disorder, as risk factors of stroke.

2) Effect of treatment of sleep-wake disorders on prevention of stroke.

3) Frequency of sleep-wake disorders as a consequence of stroke.

4) Outcome of sleep-related breathing disorders and possible treatment effects of sleep-wake disorders in patients with acute stroke.

The task force did not consider studies in subarachnoid haemorrhage and excluded studies on less common sleep-wake and circadian rhythm disorders and studies assessing their association with stroke for practical reasons.

These topics were organised in 13 research questions according to the PICO format (table 1 and supplementary material)

A systematic literature search was performed between March and July 2017. A final update search was performed in January 2019. Searches for each research question were performed applying a stepwise

TABLE 1 Research questions

\section{Pre-stroke phase}

1.1. Causation

1.2. Therapy

2.1. Causation

2.2. Therapy

2.3. Causation

2.4. Therapy

Post-stroke phase

3.1. Prevalence

3.2. Prognosis

3.3. Therapy

4.1. Prevalence

4.2. Therapy

4.3. Prevalence

4.4. Therapy
Is SDB an independent risk factor of stroke?

Does treatment of SDB prevent stroke?

Is insomnia an independent risk factor of stroke?

Does treatment of insomnia prevent stroke?

Is RLS/PLMS an independent risk factor of stroke?

Does treatment of RLS/PLMS prevent stroke?

What is the frequency of SDB in stroke patients?

Does SDB affect mortality and outcome after stroke?

Does treatment of SDB have any impact on mortality and outcome after stroke?

Is the frequency of insomnia increased in stroke patients?

Does treatment of insomnia have any impact on mortality and outcome after stroke?

Is the frequency of RLS/PLMS increased in stroke patients?

Does treatment of RLS/PLMS have any impact on mortality and outcome after stroke?

SDB: sleep-related disordered breathing; RLS: restless legs syndrome; PLMS: periodic limb movement in sleep. 
TABLE 2 Sleep disorders as independent risk factors and modulators of stroke outcome

Evidence

Statement

\begin{tabular}{|c|c|c|}
\hline \multicolumn{3}{|l|}{ Sleep disorders as risk factors for stroke } \\
\hline Sleep disordered breathing & $9 \mathrm{SR} / \mathrm{MA}, 14$ studies & Severe OSA doubles the risk of stroke \\
\hline Insomnia & 2 SR/MA, 2 studies & Uncertain effect of insomnia on risk of stroke \\
\hline Treatment of insomnia & 3 studies & Treatment with BDZ/BDZR may increase the risk of stroke \\
\hline RLS/PLMS & 3 SR/MA, 2 studies & RLS does not/PLMS may increase the risk of stroke \\
\hline Treatment of RLS/PLMS & None & No statement can be made \\
\hline Treatment of sleep disordered breathing & 2 SR/MA, 9 studies & CPAP is feasible in stroke patients and may improve outcome \\
\hline Insomnia & 8 studies & No statement can be made \\
\hline Treatment of insomnia & 1 study & No statement can be made \\
\hline RLS/PLMS & 1 SR/MA, 8 studies & RLS may be be associated with a less favourable outcome \\
\hline Treatment of RLS/PLMS & None & No statement can be made \\
\hline
\end{tabular}

Results of a systematic review performed between March and July 2017 and a final update search performed in January 2019. SR/MA: systematic reviews/meta-analyses; OSA: obstructive sleep apnea; CPAP: continuous positive airway pressure; BDZ/BDZR: benzodiazepines and benzodiazepine-related drugs; RLS: restless limb syndrome; PLMS: periodic limb movements in sleep. "Studies" refers to primary studies published after the SR/MA.

hierarchical approach: at first, possible systematic reviews and meta-analyses were searched starting from 1990. Then, 1) in the case of retrieval of systematic reviews or meta-analyses, primary studies with proper design were searched setting the time limit at the end of the systematic review search update; 2) in the case of absence of systematic reviews or meta-analyses, primary studies were searched starting from 1990.

Published studies were identified from the National Library of Medicine's MEDLINE database and Elsevier's EMBASE database. Specific search strategies used a combination of exploded terms and free text, using concepts regarding sleep disorders, stroke and treatment for sleep disorders when needed (refer to the supplementary material for the detailed search strategies). All abstracts or full papers were reviewed independently by two task force members with expertise in methodology, to identify potentially relevant studies and to assess studies for inclusion. The studies included were assessed by clinical experts in the relevant topics. The inclusion criteria for eligible studies are reported in detail in the supplementary material.

The quality of evidence of each study was classified according to topic domain and type of publication (systematic review or primary studies). Systematic reviews were assessed using the criteria from the AMSTAR checklist [40]. According to the topic, primary studies were assessed with the Classification of Evidence Schemes of the Clinical Practice Guideline Process Manual of the American Academy of Neurology [41]. Briefly, each study is graded according to its risk of bias from class I (highest quality) to class IV (lowest quality).

Statements for each research question were developed by each group of experts, with final consensus by the whole task force. The statements aim to provide an overview of the literature and current practice. They do not make recommendations for clinical practice. The questions of the scenarios having no or few evidence were considered to identify research gaps and direct future research projects.

A total of 12870 studies was evaluated for possible inclusion, full texts of 445 studies were assessed and 88 studies met the inclusion criteria (figures e1-38). A descriptive summary of the included studies with details about study design, number and characteristics of enrolled patients, intervention(s) and comparator(s), outcomes and results are provided in the supplementary tables.

\section{Results}

The main results are presented in table 2 .

\section{SDB and risk of stroke}

\subsection{Is SDB an independent risk factor for stroke?}

The possible role of OSA as a risk factor for stroke has been highlighted since the first studies using snoring as a surrogate marker of OSA [42]. According to a recent meta-analysis, habitual snoring, defined 
as occurring at least 3 nights per week, carries a 25\% additional risk for stroke compared to non-snorers [43]. However, snoring is a symptom that can occur in the absence of respiratory events during sleep, and thus objective measurement of SDB is an essential part of stroke risk evaluation.

\subsubsection{Results of literature search}

Systematic reviews: Nine studies were included [44-52]. The original studies included in the systemic reviews were of class I and II quality.

Primary studies: 14 studies of class I and II quality were included. Five studies explored the association of SDB and incident stroke in the general population [53-56]. Five studies evaluated prospectively [57-59] or retrospectively $[60,61]$ the incidence of stroke or cardiovascular events in patients studied for suspected OSA. Three studies compared cardiovascular outcomes in patients with atrial fibrillation according to presence/absence of OSA [62-64]. A retrospective study assessed incident stroke in patients with previously diagnosed OSA and end-stage renal disease [65]. A retrospective study analysed the incidence of stroke in community-dwelling men who underwent comprehensive sleep studies [66].

\subsubsection{Overview of the evidence}

The results of six systemic reviews with meta-analyses show that OSA approximately doubles the risk for stroke (relative risk (RR) ranging from 2.02 to 2.24) in untreated OSA patients over a follow-up period of 3-10 years (table e1) [44-46, 48, 52, 60]. These results stem from primary studies, the majority of which adjusted for confounding factors (age, sex, body mass index, smoking, alcohol consumption, hypertension and diabetes). These results were also confirmed in the elderly subgroup [50]. However, in patients with percutaneous coronary intervention no significant trend was found (table e2) $[49,51]$.

The incidence of stroke in OSA patients has been evaluated based on analysis of large databases. Two studies reported an increased risk, especially in young to middle-aged OSA patients [53, 54]. In community dwelling elderly men, the severity of nocturnal hypoxaemia rather than AHI appeared to carry a significant risk, especially in patients who experienced a fatal stroke during follow-up [66]. A relatively small population study confirmed an increased risk for incident stroke in patients with moderate-severe OSA [55].

The only prospective study reported a six-fold risk of experiencing stroke during follow-up in women with untreated OSA, especially in patients $<65$ years of age [53]. Two prospective studies in patients with coronary artery disease reported an increased risk for major adverse coronary or cerebrovascular event, but did not analyse stroke data specifically, likely due to small sample size [58, 59].

Retrospective studies in OSA patients yielded variable results. The study by KenDZERSKA et al. [60] reported that stroke in OSA was significantly associated with gender, age, hypertension and history of previous stroke, but not with OSA severity assessed by AHI. The only sleep variables associated with stroke were total sleep time and frequency of nocturnal awakenings. An increased risk for all-cause and cardiovascular mortality, including stroke, was found in OSA patients, but specific data on stroke were not reported [61].

Three studies examined the risk of stroke in patients with OSA and atrial fibrillation. Two studies reported a four-fold risk of stroke in OSA patients $[62,63]$, and a positive relationship between such risk and OSA severity [63]. Conversely, in elderly subjects with atrial fibrillation, OSA was not associated with increased cardiovascular risk, although OSA patients showed an increased risk for hospitalisation [64]. Finally, in a retrospective study of patients with end-stage renal disease followed for 1.6 years, OSA exerted a protective effect against mortality and ischaemic stroke or myocardial infarction (table e3) [65].

\subsubsection{Statements}

1) Untreated severe OSA doubles the risk for incident stroke.

2) Such risk appears especially relevant in young to middle-aged patients, without differences between men and women.

3) Evidence on OSA-associated risk for stroke in patients with coronary artery disease or atrial fibrillation is still insufficient, but available data suggests an increased risk in these populations, with the possible exception of elderly patients.

\subsubsection{Recommendation for future research}

Future research needs to focus on the identification of subgroups of OSA patients who may experience a high risk for incident stroke, especially regarding age, gender, comorbidities (e.g. renal failure, atrial 
fibrillation) severity of OSA based on AHI, or hypoxaemia. Development of biomarkers of increased risk would be clinically desirable, in order to implement stronger preventive actions. The mechanisms responsible for the increased risk and studies on the types of stroke occurring more frequently in OSA patients should be defined.

\subsection{Does treatment of SDB prevent stroke?}

Continuous positive airway pressure (CPAP) therapy is well recognised to abolish obstructive events during sleep, and substantially improve their consequences, especially daytime sleepiness, neurocognitive deficits and driving performance. While earlier observational studies demonstrated improved cardiovascular outcomes with CPAP therapy, more recent randomised controlled trials (RCTs) have shown no improvement $[67,68]$. Thus, the potential benefit of CPAP therapy in preventing stroke is unproven.

\subsubsection{Results of literature search}

Systematic reviews: Four studies were included [69-72]. The original studies included in the systematic reviews were of class I or II quality.

Primary studies: Four studies of class 3 or 4 quality were included. Two studies assessed CPAP [73, 74]. Two studies assessed the effect of uvulopalatopharyngoplasty (UPPP) [75] or tracheostomy [76].

\subsubsection{Overview of the evidence}

KHAN et al. [72] included seven RCTs, with SAVE having the highest weight in the meta-analysis [68]. CPAP treatment was compared with no active intervention in six RCTs; only one trial compared it with nocturnal supplemental oxygen [77]. Stroke incidence was not affected by CPAP treatment in the overall population or after the exclusion of low CPAP adherence trials and after the exclusion of SAVE trial. Sensitivity analysis for CPAP treatment at an average of $\geqslant 4 \mathrm{~h}$ per night versus matched trial controls that never used CPAP revealed a significant risk reduction in stroke incidence $\left(\mathrm{p}=0.01, \mathrm{I}^{2}=0 \%\right)$ (RR $0.56,95 \%$ CI 0.37-0.84). Risk of bias was identified in RICCADSA (table e4) [77-80].

ABUZAID et al. [69] included four RCTs with one of them not showing up in the forest plot despite assessing stroke outcome [79]. All these trials are part of meta-analysis reported by KHAN et al. [72]. CPAP treatment was not associated with a reduction in the incidence of stroke ( $\left.\mathrm{p}=0.86, \mathrm{I}^{2}=0 \%\right)$ (RR $1.01,95 \%$ CI $0.73-1.38$ ) or transient ischaemic attack (TIA) ( $\left.\mathrm{p}=0.24, \mathrm{I}^{2}=30 \%\right)$ (RR 1.36, 95\% CI 0.69-2.68). All trials were deemed to have low risk of bias and high quality body of evidence for the outcomes. The work reported by PARRA et al. $[78,81]$ is included with the 2-year rather than the 5 -year available outcomes (table e4).

KIM et al. [71] included one RCT, five cohort and two administrative health data studies. They did not show any effect of CPAP treatment on stroke incidence. The RCT had limited power to assess the effect of CPAP on stroke separately and the administrative studies had critical issues of validity and bias. A meta-analysis of three of the cohort studies showed $73 \%$ overall stroke risk reduction with CPAP; non-significant heterogeneity $\left(\mathrm{p}=0.46, \mathrm{I}^{2}=0 \%\right.$ ) ( $\mathrm{RR} 0.27,95 \%$ CI $0.14-0.53$ ) [82-84]. Poor adherence to CPAP was recorded in all but one studies [82]. The high vascular recurrence rate in this population raises concerns on the general validity of the results (table e4) [82].

Halle et al. [70] have reviewed the effect of the surgical treatment of OSA on cardiovascular outcomes. Only two studies, amongst the 33 included, reported stroke as an outcome; one was assessing UPPP and another was assessing tracheostomy (table e4) $[75,76]$.

UPPP was associated with a significant decrease in the incidence of ischaemic $(0.30 \%$ versus $1.76 \%$, $\mathrm{p}<0.0001)$ and haemorrhagic $(0.07 \%$ versus $0.47 \%, \mathrm{p}<0.0001)$ stroke; relative risk reduction after adjustments for age, sex, low income, medical comorbidities and medication was 0.41 (95\% CI $0.27-0.62$ ) and 0.53 (95\% CI 0.38-0.73), respectively [75]. Limitations of that study include the relatively small number of older patients, unknown OSA severity (insurance reimbursement claims) and absence of data on possible CPAP treatment in the non-UPPP treated population. Thus, the task force considered the quality of the study insufficient to allow any statement (table e5).

Tracheostomy compared to the conservative management group of weight-loss recommendation was associated with reduced vascular morbidity $(\mathrm{p}=0.046$, chi square $=3.98)$ and a smaller increase in the overall prevalence of ischaemic infarcts (from $9.8 \%$ to $11.0 \%$ versus $5 \%$ to $10.2 \%$ ) in the 7 -year follow-up period; it is unclear whether the latter was statistically significant [76]. This was a retrospective analysis of prospectively followed up patients from 1972 to 1980 (table e5).

Catalan-Serra et al. [73] examined the incidence of stroke in OSA patients with CPAP treatment in a prospective, observational cohort from Spain. In the 6-year median follow-up, OSA with AHI $\geqslant 30$ events 
per $\mathrm{h}$ and CPAP not prescribed or compliance $<4 \mathrm{~h}$ per day had significantly higher cumulative incidence of stroke compared with the reference group of individuals with AHI < 15 events per h (log-rank test, 11.87, $\mathrm{p}=0.001$; HR 3.42, 95\% CI 1.37-8.52) after adjustments for age, body mass index, hypertension, sex, smoking habit, Epworth Sleepiness Scale and atrial fibrillation (table e5).

Wu et al. [74] examined the effect of CPAP treatment in a retrospective cohort of OSA patients with coronary artery disease and recent percutaneous coronary intervention. In a 5-year mean follow-up period, there was no impact of CPAP treatment on stroke incidence with the trial recording a small number of cerebrovascular events (table e5).

\subsubsection{Statements}

1) Observational cohort studies suggest that CPAP treatment is associated with a reduced risk of stroke in patients with OSA, but results are very variable.

2) In meta-analyses of RCTs, CPAP treatment is not associated with stroke risk reduction in OSA patients; however, patients adherent to CPAP therapy ( $>4 \mathrm{~h}$ per day) may benefit.

3) There is insufficient evidence on other treatment options than CPAP.

\subsubsection{Recommendations for future research}

Further RCTs are needed to assess the effect of CPAP treatment with good compliance ( $>4 \mathrm{~h}$ per day) on stroke risk reduction, especially in subgroups with sleepiness, high risk profile and with severe OSA. Other OSA treatment modalities, such as mandibular advancement devices, also need to be addressed in RCTs.

\section{Insomnia, RLS/PLMS and risk of stroke}

2.1. Is insomnia an independent risk factor of stroke?

Only in recent years, insomnia has been found to be associated with cardiovascular and metabolic comorbidities, especially when linked with objective short sleep duration $[85,86]$. Uncertainties remain regarding the potential risk association for stroke, because of limitations including the variability in diagnostic criteria for insomnia and the confounding influence of comorbidities.

\subsubsection{Results of literature search}

Systematic reviews: Two systematic reviews were found. One systematic review included 15 prospective studies (cohort, sub-cohort and population-based studies) [87, 88]. The original studies included in the systematic review by $\mathrm{He}$ et al. [87] were of class I quality.

Primary studies: Two case-control studies (nested in an administrative cohort) of class III quality were included $[89,90]$.

\subsubsection{Overview of the evidence}

The systematic review by $\mathrm{HE}$ et al. [87] showed that insomnia increases the risk of future cardio- or cerebrovascular events with an odds ratio below 1.3, similar to most studies linking long and short sleep with stroke [26, 32]. However, when considering only studies with stroke as outcome, data were insufficient to support a link between insomnia and stroke. In fact, only two studies in this meta-analysis assessed the relationship between insomnia and stroke [91, 92]. In the study of HeLBIG et al. [91], there were 917 strokes observed in a cohort of 17604 subjects followed for a mean period of 14 years. After adjusting for other risk factors, symptoms of insomnia and short sleep duration were not predictive of stroke in either sex. In the study of WeSTERLUnd et al. [92], including 41192 adults with a follow-up of 13.2 years, 1685 strokes were observed. The authors found that insomnia was unrelated to risk of overall cardiovascular events. However, short sleepers $(\leqslant 5 \mathrm{~h})$, with frequent insomnia symptoms had an increased risk (HR 1.26-1.39) (table e6).

The systematic review by Kwok et al. [88] did not find an association between poor sleep quality and stroke outcome (table e6).

Other two studies based on administrative data, both from Taiwan (Wu et al. [89] and Hsu et al. [90]), reported a higher risk for stroke in patients diagnosed to have insomnia according to ICD codes (adjusted HR ranging from 1.54 to 1.85 , statistically significant) (table e7).

\subsubsection{Statements}

1) Insomnia slightly increases the risk for cardiovascular events, but risk for stroke is uncertain. 
2) Prospective studies evaluating the association of insomnia with stroke risk are scarce and all available studies are based on subjective measures to define insomnia, thus reducing the accuracy of exposure identification.

\subsubsection{Recommendations for future research}

There is a need for more prospective studies, using standardised and validated self-assessment questionnaires, and objective measures for evaluating insomnia severity. Repeated measures of sleep issues are required, as insomnia symptoms may change over time. Information about the presence of comorbidity with other sleep disorders, and data regarding treatment of insomnia during the follow-up period, should be included.

\subsection{Does treatment of insomnia prevent stroke?}

Chronic use of benzodiazepines and related drugs (BDZR, such as zopiclone and zolpidem) has been linked in several studies with increased risk of impaired cognitive function, dementia and mortality [93-96]. However, the effect of insomnia treatment on stroke risk has been rarely assessed.

\subsubsection{Results of literature search}

Systematic reviews: None were identified.

Primary studies: Three studies (two of class II quality, one of class III) were included in our review of the evidence [97-99].

\subsubsection{Overview of the evidence}

ZHU et al. [98] observed a significant decrease in ischaemic stroke risk in users of gamma-aminobutyric acid (GABA) agonists. In contrast, the use of benzodiazepines (BDZ) failed to improve the risk of ischaemic stroke. A significant decrease in ischemic stroke risk was observed among patients using non-BDZ (adjusted OR 0.48, 95\% CI 0.32-0.72). BDZ use was not associated with ischaemic stroke (adjusted OR 1.25, 95\% CI 0.91-1.72). Adjusted odds ratios in patients using non-BDZ were 0.75 (95\% CI $0.39-1.46$ ) for $0-5$ years, 0.44 (95\% CI $0.25-0.77$ ) for 5-10 years, and 0.38 (95\% CI $0.16-0.90)$ for $>10$ years. The risk of ischaemic stroke decreased progressively with duration of non-BDZ use $(\mathrm{p}<0.001)$ [98].

The second study retrospectively compared the risk of stroke in patients with $(n=38663)$ or without BDZ therapy $(\mathrm{n}=38671)$. Compared to non-treated patients, those with a lower annual dosage of BDZ $(<1 \mathrm{~g})$ or duration ( $<30$ day) had a significantly lower risk of stroke in the elderly group, while patients with a higher annual dosage $(\geqslant 4 \mathrm{~g})$ or duration $(\geqslant 95$ day) of $\mathrm{BDZ}$ use had a higher risk of stroke in all age groups [97].

TAIPALE et al. [99] investigated the risk of ischaemic, haemorrhagic or any stroke associated with incident BDZR use in 45050 community-dwelling individuals with Alzheimer's disease. Compared with non-use, BDZR use was associated with an increased risk of any stroke (adjusted HR 1.21, 95\% CI 1.04-1.40) and ischaemic stroke (adjusted HR 1.21, 95\% CI 1.02-1.44), but the association between BDZR use and haemorrhagic stroke did not reach statistical significance (adjusted HR 1.26, 95\% CI 0.91-1.74). The use of BDZR was associated with a similar risk as BDZ use.

\subsubsection{Statement}

Treatment of insomnia with BDZ/BDZR is linked with an increased risk of cognitive dysfunction, dementia and mortality and possibly also stroke, especially in high dosage and long-term use. This effect may be related also to an indication bias: patients who are in a worse general or neurological condition suffer more frequently from insomnia and may receive BDZ/BDZR more frequently.

\subsubsection{Recommendations for future research}

There is a need for systematic and prospective studies on the effect of BDZ/BDZR and other insomnia treatments (including non-pharmacological approaches) on the risk of stroke. Standardised and validated questionnaires for evaluating insomnia and data on possible comorbidities should be considered.

\subsection{Is RLS/PLMS an independent risk factor of stroke?}

RLS with short total sleep time and PLMS with sleep fragmentation may cause a sympathetic hyperactivity, activate the hypothalamic pituitary adrenal axis, and increase the levels of pro-inflammatory cytokines and circulating catecholamines $[23,100]$, which may favour hypertension and stroke. 


\subsubsection{Results of literature search}

Systematic reviews: Two systematic reviews on RLS including 18 studies and eight studies, respectively, were considered [101, 102]. For PLMS there is a recent systematic review that included five studies [103]. The original studies included in the systematic review were of class II and III quality.

Primary studies: Two additional primary studies of class II [104] and class IV quality [105] were identified.

\subsubsection{Overview of the evidence}

The first systematic review on RLS concluded that available evidence on RLS as a prognostic factor for incident cardiovascular events and all-cause mortality was limited and inconclusive; two of six cohort studies of moderate quality found an association between RLS and stroke [101]. However, none of the primary studies included in this review met class I criteria and the remaining studies were of limited quality. A major problem of these studies is the diagnosis and classification of RLS (table e9).

The most recent systematic review with meta-analysis on RLS did not provide any evidence for an increased stroke risk in RLS [102]. In the unadjusted analyses of prospective observational studies, RLS patients were found to have significantly higher risk for cerebrovascular ischaemia $(p=0.01)$ and all-cause mortality $(p=0.04)$ compared to controls. However, in the analysis adjusted for potential confounders, RLS patients were found to have a higher risk only for all-cause mortality (adjusted HR 1.52, p=0.002) (table e9).

The meta-analysis on PLMS included 9823 patients with PLMS and 9416 controls from five studies [103]. The authors performed meta-regression and subgroup meta-analysis to detect the potential confounding factors. An increased prevalence of stroke in patients with PLMS compared to controls (OR 1.267, within 8 years of a diagnosis of PLMS) was found (table e9).

In the first additional primary study, Winkelman et al. [104] published data about the MrOS Sleep Study that included 2823 community-dwelling men 65 years or older. RLS was identified by self-report of a physician diagnosis of RLS. A periodic limb movement of sleep index (PLMI) was derived from unattended in-home polysomnography (PSG). Incident cardiovascular events were centrally adjudicated during 8.7 \pm 2.6 years of follow-up. RLS and PLMI were not associated with stroke (table e10).

The second additional primary study is a case-control study $(\mathrm{n}=487$ primary RLS patients and 354 controls) that reported no significant association between RLS and stroke [105].

\subsubsection{Statements}

1) Current evidence does not suggest an increased risk of stroke in patients with RLS.

2) PLMS may represent an independent risk factor of stroke.

\subsubsection{Recommendations for future research}

Long-term controlled cohort studies using strict internationally accepted definitions and controlling of comorbidities and other confounders to the presence of RLS are needed. The concomitant evaluation of PLMS in RLS patients may be needed but represents at the same time a major logistical challenge.

\subsection{Does treatment of RLS/PLMS prevent stroke?}

Considering the possible link between PLMS and stroke, treatment of RLS patients (presenting PLMS in $70-80 \%$ of cases) could be beneficial in reducing stroke risk.

\subsubsection{Results of literature search}

Systematic reviews: No studies were found.

Primary studies: No studies were found.

\subsubsection{Overview of the evidence}

No data to be discussed.

\subsubsection{Statements}

Based on the lack of evidence, no statement can be made.

\subsubsection{Recommendations for future research}

Long-term studies on the effect of RLS/PLMS treatment on stroke are required. Drugs with different impact on sleep fragmentation or PLMS should be compared. 


\section{Frequency of SDB after stroke and its impact on outcome}

\subsection{What is the frequency of SDB in stroke patients?}

Early studies [106, 107] suggested a high frequency of SDB after stroke, which has been confirmed in many subsequent more recent studies. Since SDB is frequent in the general population and stroke can lead to "de novo" SDB, it remains uncertain for individual patients whether SDB is pre-existing or a consequence of stroke [31]. The form of SDB (central sleep apnoea or OSA) and its evolution after stroke have been only rarely assessed. In addition, the differentiation between obstructive and central events was unclear/questionable in most studies.

\subsubsection{Results of the literature search}

Systematic reviews: Three systematic reviews/meta-analyses on the frequency of SDB in stroke were retrieved $[8,108,109]$. The original studies included in the systematic reviews were of quality ranging from class I to III.

Primary studies: Six additional primary studies of class II or III quality were identified [110-115].

\subsubsection{Overview of the evidence}

The systematic review by JoHnson et al. [108] included 29 studies involving 2343 patients with TIA, ischaemic or haemorrhagic stroke. The frequency of SDB with AHI $>10$ was 63\% (95\% CI 58-68\%) and with AHI $>30$ was $29 \%$ (95\% CI $21-37 \%$ ). Only $7 \%$ of the SDB was primarily central apnoea. There was no significant difference in SDB prevalence by event type, timing after stroke, or type of monitoring. Males had a higher percentage of AHI $>10$ than females (65\% compared to $48 \%$; $=0.001$ ). Patients with recurrent strokes had a higher percentage of AHI $>10$ than initial strokes ( $74 \%$ compared to 57\%; $\mathrm{p}=0.013$ ). Patients with cardioembolic aetiology had a lower percentage of SDB than other aetiologies (table e11).

The systematic review by Dong et al. [8] included a total of 37 studies. 18 studies assessed patients within 7 days, 10 after 7-28 days, and three studies over 28 days after stroke. The frequencies for the different AHI cut-offs were found: AHI >5 in 70.4\% (95\% CI 62.1-78.7\%), >20 in 39.5\% (95\% CI 31.6-47.4\%), and $>30$ in $30.1 \%$ (95\% CI 23.1-37.0\%) (table e11).

The meta-analysis by SEILER et al. [109] included a total of 89 studies where at least 10 patients were assessed, and full PSG or portable device were used for diagnosis (table e11). 54 studies assessed patients $<1$ month, 23 studies 1-3 months, and 12 studies $>3$ months after stroke. The distribution of stroke types was as follows: ischaemic stroke 5275, haemorrhagic stroke 302, TIA 405, undefined 1188 . The frequencies for the different AHI cut-offs were found: AHI $>5$ in $70-90 \%$ (95\% CI 66.6-74.8\%), AHI $>20$ in $40 \%$ (95\% CI 33.45-46.85\%) AHI >30 in 30\% (95\% CI 24.4-35.5\%). The mean AHI was 26 (95\% CI 21.7-31.2, $\mathrm{n}=44$, I2 24\%); the mean obstructive apnoea index was 12 (95\% CI 6.5-21.1, n=12, I2 32\%), the mean central apnoea index was 5 (95\% CI 2.8-10.0, $\mathrm{n}=13, \mathrm{I} 2 \mathrm{12} \%)$, and all the other events were hypopnoeas, adding up to the overall mean AHI. The frequency of SDB did not differ depending on the interval between stroke and sleep study; however, three studies providing longitudinal observations of SDB evolution suggested improvement over time. There was no difference whether SDB was diagnosed with PSG or portable device study. Few studies assessed risk factors/predictors of SDB in stroke patients. Six new studies, published after the most up-to-date systematic review, did not add new data on this topic (table e12) [95, 110-115]. Most studies published did not find any relationship between topography of stroke and frequency/severity of SDB, although few reports suggested an association between central SDB and supratentorial, and obstructive SDB and infratentorial stroke [31].

\subsubsection{Statements}

1) The prevalence of SDB is high in stroke patients; about $30 \%$ of stroke patients present with severe SDB $(\mathrm{AHI}>30)$.

2) Portable cardiorespiratory polygraphy is sufficient to assess the presence/severity of SDB in stroke patients in clinical practice.

3) Predictors of SDB in stroke patients have not been adequately assessed.

4) The link between stroke characteristics (type, severity, topography, aetiology) and severity or type of SDB is insufficiently understood.

5) The evolution of SDB over time remains uncertain.

\subsubsection{Recommendations for future research}

New tools are needed for the assessment of severity, type and implications of SDB post-stroke. Predictors of SDB in stroke patients should be determined. More longitudinal studies are needed to assess the 
evolution of severity and optimum time for treatment interventions. The impact of stroke type, severity and topography could be of clinical/pathophysiological interest but is not well known. The significance of different subtypes of central sleep apnoea is poorly known.

\subsection{Does SDB affect mortality and outcome after stroke?}

Untreated severe OSA is associated with a risk of incident stroke. The risk is especially increased in young to middle-aged patients and possibly associated with other cardiovascular comorbidities in OSA patients. As incidence of severe OSA is high in stroke patients, the question arises if SDB might influence survival and outcome in stroke patients.

\subsubsection{Results of the literature search}

Systematic reviews: The literature search revealed two systematic reviews $[116,117]$. The original studies included in the systematic reviews were of quality ranging from class I to IV.

Primary studies: Five primary studies of class III quality $[111,113,114,118,119]$ were included.

\subsubsection{Overview of the evidence}

One systemic review included seven cohort studies and one cross-sectional study with 47-174 patients [116]. The systematic review with meta-analysis of 13 hospital-based cohort studies focussed on stroke (five studies, 860 patients) and mortality (11 studies, 930 patients) [117]. The mean ages in both reviews were between 56 and 79 years; $41-87 \%$ of subjects were males. The reviews were limited by different levels of severity, heterogeneous adjustments for confounders and a variety of follow-up periods. The advantages of the systematic review with meta-analysis were the prospective character of the cohort studies, the large sample size and the missing evidence of significance between study heterogeneity and of change in sensitivity analyses for the outcomes. The reviews showed consistently that SDB increases the risk of recurrent stroke and all-cause mortality. However, effects of SDB on the neurological outcome, e.g. measured as modified Rankin Scale score, were not reported (table e13).

New studies, published after the most up-to-date systematic review, did not find an independent association between SDB and mortality [111, 114, 119]; however, three of them $[113,114,118]$ found an association of SDB with a worse neurological outcome (e.g. modified Rankin Scale score, Bartel Index, early neurological deterioration) (table e14).

\subsubsection{Statements}

1) OSA is a risk factor for recurrence of stroke or TIA.

2) OSA in stroke patients may be associated with an increase in all-cause mortality [27] and worsen the neurological outcome.

\subsubsection{Recommendations for future research}

Future studies should include larger sample sizes, adjust appropriately for potential confounders, and evaluate neurological outcomes in greater detail, including cognitive functions. Large, long-term prospective studies are required in patients with SDB but without previous cerebrovascular events.

\subsection{Does treatment of SDB have any impact on mortality and outcome after stroke?}

While SDB and, especially, OSA is common in stroke patients and associated with an increased risk of recurrent stroke and all-cause mortality, the impact of treating SDB on neurological recovery and cardiovascular morbidity and mortality has only recently been explored.

\subsubsection{Results of the literature search}

Systematic reviews: The search revealed two updated systematic reviews with meta-analyses using CPAP or non-invasive ventilation (NIV) as treatment for SDB [120, 121].

Primary studies: A further two class II quality studies on the impact of CPAP on measures of vascular risk were published after the systematic search finished and were included $[122,123]$.

Seven primary studies of class II to IV quality using other interventions were included: one RCT using positional therapy [124], six proof-of concept studies or retrospective analyses using adaptive servo-ventilation, expiratory positive airway pressure, oxygen, transnasal insufflation, oropharyngeal muscle exercise or mirtazapine [125-130]. 


\subsubsection{Overview of the evidence}

The systematic review of Tsivgoulis et al. [120] focused on the acute stroke setting and included four RCTs using CPAP and one prospectively matched observational cohort study with NIV. The mean decrease in the National Institutes of Health Stroke Scale (NIHSS) score during the initial $(\leqslant 30)$ days after acute ischaemic stroke was greater in NIV treated patients in comparison to controls (standardised mean difference (SMD) $0.38,95 \%$ CI $0.11-0.66 ; \mathrm{p}=0.007$ ). There was a risk for detection and performance bias and the authors concluded that NIV seems to be associated with greater short-term neurological improvement in acute ischaemic stroke patients with OSA, but acknowledged the limitations of the included primary studies (table e15).

The second meta-analysis by BRILL et al. [121] included 10 RCTs using CPAP ( $\mathrm{n}=564)$ to improve stroke outcomes in acute and chronic stroke survivors with OSA (table e15). The combined analysis of the stroke scales (NIHSS and Canadian Neurological Scale) showed an overall improvement with CPAP (SMD 0.5406, 95\% CI $0.0263-1.0548$ ) but were limited by a considerable heterogeneity across the studies. The results of the neurocognitive tests, depression scores and sleepiness were inconsistent across the trials. The adherence to CPAP was acceptable once the treatment was tolerated (mean CPAP usage across the trials $4.53 \mathrm{~h}$ per night, 95\% CI 3.97-5.08 h per night). All trials struggled with recruitment and overall acceptance of CPAP which partially limits the power of the trials and the effectiveness of the intervention. The odds ratio of dropping out with CPAP was 1.83 (95\% CI 1.05-3.21; $\mathrm{p}=0.033$ ).

Recurrent cardiovascular events and mortality have been assessed in only one RCT $(n=140)$ and revealed a longer time to the next cardiovascular event (14.9 versus 7.9 months), but no difference in the overall cardiovascular event-free survival or all-cause mortality over 2 years [81]. However, at 5 -year follow-up, CPAP was associated with a higher cardiovascular survival rate compared to the control group [78]. A non-randomised observational study $(\mathrm{n}=166)$ showed that an AHI $>20$ and intolerance of CPAP has a hazard ratio of 2.87 (95\% CI 1.11-7.71) for a non-fatal cardiovascular event [82]. BRAVATA et al. [122] reported no difference in neurological status and functioning, when comparing CPAP therapy with no CPAP in the intention-to-treat analysis. However, CPAP-compliant patients demonstrated improved stroke outcome as assessed by the NIHSS and modified Rankin Scale scores. GuPTA et al. [123] reported no difference of vascular event incidence (primary outcome) at 12 -month follow-up (3.33\% in the CPAP group $15 \%$ in the non-CPAP group, $\mathrm{p}=0.23$ ). Modified Rankin Scale score improvement by $\geqslant 1$ (secondary outcome) was found in significantly more patients in the CPAP group than in the non-CPAP group ( $53 \%$ versus $27 \%, \mathrm{p}=0.03$ ) (table e16).

There is very little or no evidence on the effect of specific SDB treatment alternatives, such as oropharyngeal muscle exercise [130], positional therapy [124] or mandibular advancement devices, or other positive airway pressure modes [127-129], oxygen [125] or pharmacotherapy [126], on stroke outcomes, cardiovascular events or mortality (table e17).

\subsubsection{Statements}

1) Current evidence suggests that CPAP is feasible in stroke survivors with OSA and may improve neurological recovery, sleepiness and depressive symptoms. It further suggests that treatment of post-stroke OSA with CPAP should be included in an integrated management of multiple risk factors such as anticoagulation for atrial fibrillation, control of hypertension and dyslipidaemia, exercise and weight reduction.

2) Acceptance of CPAP in the acute stroke setting within trials was mostly limited, but once accepted, compliance can be satisfactory.

3) There is insufficient evidence to permit statements on the use of other treatment modalities to influence stroke outcome.

\subsubsection{Recommendations for future research}

Future research needs to focus on stroke specific multidisciplinary educational programmes for patients, staff and caregivers to enable stroke survivors to be more adherent to CPAP (mask-fitting, handling of the machine). Other research areas include the identification of patients who might have the highest likelihood of benefiting from the treatment, the optimal timing of treatment initiation and the use of meaningful and comparable neurocognitive and neurofunctional endpoints in sufficiently powered trials.

\section{Frequency of insomnia and RLS/PLMS after stroke and their impact on outcome} 4.1. Is the frequency of insomnia increased in stroke patients?

Insomnia is highly prevalent in the general population and stroke can lead "de novo" to insomnia [27]. Accordingly, a recent meta-analysis of sleep electroencephalogram studies showed that stroke patients have a poorer sleep than controls, in term of sleep efficiency, total sleep time and wake after sleep onset [131]. 
In addition, the presence of insomnia in stroke patients has been shown to be linked with cognitive impairment, anxiety, depression (and suicidality) and to have a bad impact on functional recovery and return to work [30, 132-134]. Unfortunately, few studies addressed systematically the frequency of insomnia in stroke patients.

\subsubsection{Results of literature search}

Systematic reviews: No studies were found.

Primary studies: Eight studies of class II and III quality were included [132, 134-140].

\subsubsection{Overview of the evidence}

In a study of 277 consecutive patients assessed in the first month after onset $56.7 \%$ had complaints of insomnia and $37.5 \%$ had insomnia according to the Diagnostic and Statistical Manual of Mental Disorders (DSM) IV criteria [135]. In 18\% of patients, insomnia appeared for the first time (de novo) after brain damage (table e18).

In a study of 100 stroke patients, complaints of insomnia (as assessed by the insomnia-related items of the Hamilton depression scale) occurred in $68 \%$ of patients on admission, and in $49 \%$ at 18 months after stroke. From 2 months, symptoms of insomnia were associated independently with depression. Living alone before stroke and age were other independent predictors of insomnia (table e18) [136].

In a study of 40 stroke patients and 30 healthy controls assessed by the Sleep Habits Questionnaire, insomnia complaint was the most prevalent (37.5\% versus $6.7 \%$; $\mathrm{p}=0.007)$. Female sex (OR $11.098,95 \% \mathrm{CI}$ 1.167-105.559; $\mathrm{p}=0.036$ ) and fragmented sleep (OR 32.040, 95\% CI 3.236-317.261; $\mathrm{p}=0.003$ ) were risk factors for insomnia (table e18) [137].

In a study of 366 patients assessed 3 months after stroke, insomnia (as assessed by a seven-item questionnaire) was found in $44 \%$ of them; an association with female gender, depression and lower stroke-related quality of life was observed [132]. In a study of 215 first-time stroke patients, insomnia (as defined as presence of at least one of the four following complaints: difficulty initiating sleep, difficulty maintaining sleep, early morning awakening and non-restorative sleep) 1 month after onset was found in 59.5\%. Insomnia was found to be significantly associated with poorer physical functioning, general health and vitality scores [138]. In a study of 280 patients assessed 1 month after stroke, insomnia (as defined by DSM IV criteria) was found in $26.9 \%$ of patients and was associated with poorer functional recovery [139]. In a study of 441 patients assessed by self-report and interview at 1, 6 and 12 months after stroke, the point prevalence of insomnia (as assessed by three items of the Karolinska sleep questionnaire) at each time point in the year after stroke was stable at 30-37\% and more common in females. 58 (16\%) of all participants reported "chronic" insomnia, with symptoms at both baseline and 6 months later. At 12 months, this group was more likely to be depressed (OR 6.75,95\% CI 2.78-16.4), anxious (OR 3.31, 95\% CI 1.54-7.09), disabled (OR 3.60, 95\% CI 2.07-6.25), and not have returned to work, compared to those without insomnia over the same period (table e18) [134].

In a study of 1062 first-time stroke patients, insomnia (as defined by DSM IV criteria) was reported by $38.4 \%$. During the 6 years of follow-up, after adjusting for all confounders, insomnia was found to be associated with increased mortality (HR 1.66, 95\% CI 1.10-2.48) (table e18) [140].

Insomnia, as assessed by a variety of questionnaires and diagnostic criteria, is found in about one third of stroke patients (range 27-60\%) and is associated with female gender, depression and poorer functional outcome. Data on long-term evolution are limited but suggest that post-stroke insomnia can persist over 12-18 months (table e18).

\subsubsection{Statements}

1) The prevalence of insomnia in stroke patients is increased (at least 30\%).

2) There is a link between post-stroke insomnia, female gender and depression.

3) The evolution of insomnia over time is uncertain.

\subsubsection{Recommendation for future research}

There is a need of a prospective studies on post-stroke insomnia using standard diagnostic tools and control groups. Risk factors for post-stroke insomnia (including stroke characteristics), evolution of insomnia over time and the prognostic role of insomnia on stroke-related outcomes should also be considered. 


\subsection{Does treatment of insomnia have any impact on mortality and outcome after stroke?}

Insomnia is found in at least one third of stroke patients and has a negative impact on functional evolution and quality of life (see above). Experimental and (few) human data suggest that GABA agonists (and also GABA antagonists) may have favourable effects on stroke evolution and outcome [141-143]. However, GABA agonists and BDZR can lead in stroke patients to the re-emergence of neurological deficits [144, 145]. In addition, treatment of insomnia with BDZR increases the risk of dementia, mortality and possibly also stroke (see above). Finally, a recent meta-analysis suggested that the use of GABA receptor agonists (such as chlormethiazole or diazepam) may be detrimental in the acute phase of stroke [146]. Following single case reports only few studies on the treatment of insomnia in stroke victims were published.

\subsubsection{Results of literature search}

Systematic reviews: None were identified.

Primary studies: One class IV quality study was included [147].

\subsubsection{Overview of the evidence}

In a study of 15 patients with subacute stroke and insomnia, hypnotics were found to improve sleep but not cognition, depression or functional outcome (table e19) [147].

\subsubsection{Statement}

There are no systematic data on the effect of insomnia treatment on stroke outcome; worsening of neurological deficits with hypnotics has been reported.

\subsubsection{Recommendations for future research}

Randomised placebo-controlled trials should be conducted in patients with post-stroke insomnia. The presence of comorbidities such as depression should be taken into account. Because of higher mortality rates with most classes of psychotropic drugs, an RCT evaluating non-pharmacological approaches should also be considered.

\subsection{Is the frequency of RLS/PLMS increased in stroke patients?}

RLS/PLMS is highly prevalent in the general population and stroke can lead "de novo" to RLS and PLMS [27].

\subsubsection{Results of literature search}

Systematic reviews: A recent systematic review of three studies on PLMS was included [103]. The original studies included in the systematic review were of class II and III quality.

Primary studies: Eight studies of class II and III quality were included [148-155]. Three studies evaluated prospectively the frequency of RLS/PLMS in stroke patients [149-151].

\subsubsection{Overview of the evidence}

The meta-analysis evaluated 158 PLMS patients with stroke and 88 PLMS patients without stroke from three studies [103]: a higher PLMI was found in stroke patients (Hedges'g=0.860, mean difference: 4.43) (table e20).

GUPTA et al. [151] found a prevalence of RLS of 12\% (10\% before stroke and 2 post-stroke). Out of 346 consecutive patients in a single stroke unit (80\% males), 29 of 35 patients with pre-stroke RLS had imaging evidence for a subcortical stroke. The most significant difference between patients with subcortical stroke and those with cortical stroke was the presence of RLS before stroke $(23 \%$ versus $3 \%, \mathrm{p}<0.001)$ (table e21).

SHIINA et al. [155] found a prevalence of RLS of 7.7\% (3.3\% post-stroke) out of 104 consecutive patients in a single hospital. In a prospective study of 137 patients, LEE et al. [149] found post-stroke RLS in 12.4\% of patients (one patient with cortical and 16 patients with subcortical stroke). SCHLESINGER et al. [148] found pre-stroke RLS in $12.5 \%$ of patients (no post-stroke patients), statistically different from the $3 \%$ found in controls. In a prospective study, MEDEIROs et al. [150] found RLS in $12.5 \%$ of 97 stroke patients, all of them reported RLS before stroke. Patients with RLS had a significantly worse outcome at 3 and 12 months. In a prospective study, Boulos et al. [152] found RLS in $24 \%$ of 96 patients with TIA or stroke. The presence of RLS correlated with poorer outcome and depression. Another prospective study by Boulos et al. [153] found periodic limb movements in 50\% of 30 patients with TIA or stroke. Two other recent studies suggested an association between post-stroke RLS and pontine stroke [156, 157]. MANCONI et al. [154] reported a similar frequency of PLMS in 169 stroke patients examined by conventional PSG in both the 
acute and subacute phase and no difference with 162 controls. Noteably, stroke patients with PLMS were on a higher number of antihypertensive drugs (table e21).

GUPTA et al. [151] suggest that, out of 346 consecutive patients in a single stroke unit (80\% males), 29 of 35 patients with pre-stroke RLS had imaging evidence for a subcortical stroke. The most significant difference between patients with subcortical stroke and those with cortical stroke was the presence of RLS before stroke ( $23 \%$ versus $3 \%, \mathrm{p}<0.001)$. However, 14 of the patients had an AHI $>5$ and no information on medication prior to stroke was available. In a prospective study of 137 patients, LeE et al. [149] found RLS in one patient with cortical and 16 patients with subcortical stroke (table e21).

\subsubsection{Statements}

1) Data on the prevalence of RLS after stroke are inconclusive.

2) RLS in stroke patients is associated with subcortical/brainstem stroke and may be associated with a less favourable outcome.

3) Data on the prevalence and impact of PLMS after stroke are controversial, although PLMS in stroke patients may be more severe than in the general PLMS population.

\subsubsection{Recommendations for future research}

Larger prospective studies are needed to assess the frequency and impact of RLS and PLMS in stroke patients. Objective measures and assessment of confounding variables such as OSA should be considered.

\subsection{Does treatment of RLS/PLMS have any impact on mortality and outcome after stroke?}

Considering the impact of RLS/PLMS on cardiovascular functions and the possibility of an increased risk of stroke in patients with PLMS (see above) and of a poorer outcome in stroke patients with RLS treatment of RLS/PLMS in this context could be of interest.

\subsubsection{Results of literature search}

Systematic reviews: None were identified.

Primary studies: No study was found.

\subsubsection{Overview of the evidence}

No data are available.

\subsubsection{Statement}

Based on the lack of evidence, no statement can be made.

\subsubsection{Recommendation for future research}

Prospective studies on treatment of RLS and PLMS in stroke patients could be of interest.

\section{Concluding remarks}

This statement comes to three main conclusions. First, OSA (which affects $10-20 \%$ of the general population and $50 \%$ of stroke patients) likely increases the risk of stroke and worsens its outcome. Second, CPAP possibly has a favourable effect on both stroke risk and outcome. Third, non-apnoea SWD (which are common in both general population and stroke patients) may be associated with an increased stroke risk and a worse outcome. It is noteworthy that a recent meta-analysis published by GoTTLIEB et al. [35] suggested that long sleep duration hypersomnia and self-reported REM sleep behaviour disorder, SWD which were not analysed in our study, may also increase the risk of stroke.

Altogether, current evidence supports the hypothesis of a bidirectional link between SWD and stroke and calls for a stronger collaboration between stroke and sleep clinicians [158].

Future research should test the hypothesis that different phenotypes of SDB and OSA (e.g. with sleepiness, insomnia, or none of them) may be differently associated with various cardiovascular complications, including stroke. Overall, more studies are needed to determine the exact extent and pathophysiological base of the reported associations between SWD and stroke and to identify the best options to improve stroke prevention and outcome through sleep-related interventions.

Acknowledgements: We thank Carla Miltz for her assistance in the management of the manuscript and Romano Hönger for his assistance in the management of the task force; Elisa Baldin (Bologna, Italy) and Matthias Egger for assisting in the methodological process; Beatrice Minder and Doris Kopp for assisting in the search strategy design; Paul 
Twiddy and Jenny Emmel from the Library and Information Service, Leeds Teaching Hospitals NHS Trust for the support in literature search; and Thorsten Steiner for additional input.

Conflict of interest: C.L.A. Bassetti has nothing to disclose related to this work. W. Randerath reports grants and personal fees for lectures from Weinmann, Philips Respironics, Resmed, Inspire, Heinen and Löwenstein and Genzyme, outside the submitted work. L. Vignatelli has nothing to disclose. L. Ferini-Strambi reports personal fees for advisory board work from UCB-Pharma, Italfarmaco, Resmed, Pfizer and Vitalaire Italia, outside the submitted work. A-K. Brill has nothing to disclose. M.R. Bonsignore has nothing to disclose. L. Grote reports grants and personal fees for lectures from Resmed and Itamar, personal fees for lectures from Philips, during the conduct of the study; personal fees for lectures from AstraZeneca, outside the submitted work; in addition, L. Grote has a patent for pharmacological treatment of sleep apnoea licensed to Desitin. P. Jennum has nothing to disclose. D. Leys reports compensation paid to hospital research funds and Adrinord for trials, advisory boards and symposia, from BMS/Pfizer, Boehringer Ingelheim and Bayer, outside the submitted work; and is vice editor of the European Stroke Journal. J. Minnerup has nothing to disclose. L. Nobili has nothing to disclose. T. Tonia acts as ERS methodologist. R. Morgan has nothing to disclose. J. Kerry has nothing to disclose. R. Riha has nothing to disclose. W.T. McNicholas has nothing to disclose. V. Papavasileiou has nothing to disclose.

\section{References}

1 Feigin VL, Forounzanfar MH, Krishnamurthi R, et al. Global and regional burden of stroke during 1990-2010: findings from the Global Burden of Disease Study 2010. Lancet 2014; 383: 245-255.

2 O'Donnell MJ, Chin SL, Rangarajan S, et al. Global and regional effects of potentially modifiable risk factors associated with acute stroke in 32 countries (INTERSTROKE): a case-control study. Lancet 2016; 388: 761-775.

3 Bassetti CL, Ferini-Strambi L, Brown S, et al. Neurology and psychiatry: waking up to opportunities of sleep: state of the art and clinical/research priorities for the next decade. Eur J Neurol 2015; 22: 1337-1354.

4 Heinzer R, Vat S, Marques-Vidal P, et al. Prevalence of sleep-disordered breathing in the general population: the HypnoLaus study. Lancet Respir Med 2015; 3: 310-318.

5 Arnardottir ES, Bjornsdottir E, Olafsdottir KA, et al. Obstructive sleep apnoea in the general population: highly prevalent but minimal symptoms. Eur Respir J 2016; 47: 194-202.

6 Benjafield AV, Ayas NT, Eastwood PR, et al. Estimation of the global prevalence and burden of obstructive sleep apnoea: a literature-based analysis. Lancet Respir Med 2019; 7: 687-698.

7 Senaratna CV, Perret JL, Lodge CJ, et al. Prevalence of obstructive sleep apnea in the general population: A systematic review. Sleep Med Rev 2017; 34: 70-81.

8 Dong R, Dong Z, Liu H, et al. Prevalence, risk factors, outcomes, and treatment of obstructive sleep apnea in patients with cerebrovascular disease: a systematic review. J Stroke Cerebrovasc Dis 2018; 27: 1471-1480.

9 McNicholas WT, Bassetti CL. Sleep and breathing disorders: a multidisciplinary approach. Eur Respir Rev 2013; 22: 197-198.

10 McNicholas WT, Bassetti CL, Ferini-Strambi L, et al. Challenges in obstructive sleep apnoea. Lancet Respir Med 2018; 6: 170-172.

11 Randerath W, Bassetti CL, Bonsignore MR, et al. Challenges and perspectives in obstructive sleep apnoea: report by an ad hoc working group of the Sleep Disordered Breathing Group of the European Respiratory Society and the European Sleep Research Society. Eur Respir J 2018; 52: 1702616.

12 Ye L, Pien GW, Ratcliffe SJ, et al. The different clinical faces of obstructive sleep apnoea: a cluster analysis. Eur Respir J 2014; 44: 1600-1607.

13 Riemann D, Baglioni C, Bassetti C, et al. European guidelines for the diagnosis and treatment of insomnia. J Sleep Res 2017; 26: 675-700.

14 Lembke A, Papac J, Humphreys K. Our other prescription drug problem. N Engl J Med 2018; 378: 693-695.

15 Tobaldini E, Costantino G, Solbiati M, et al. Sleep, sleep deprivation, autonomic nervous system and cardiovascular diseases. Neurosci Biobehav Rev 2017; 74: 321-329.

16 Sofi F, Cesari F, Casini A, et al. Insomnia and risk of cardiovascular disease: a meta-analysis. Eur J Prev Cardio 2014; 21: 57-64.

17 Meng L, Zheng Y, Hui R. The relationship of sleep duration and insomnia to risk of hypertension incidence: a meta-analysis of prospective cohort studies. Hypertens Res 2013; 36: 985-995.

18 St-Onge MP, Grandner MA, Brown D, et al. Sleep duration and quality: impact on lifestyle behaviors and cardiometabolic health: a scientific statement from the American Heart Association. Circulation 2016; 134: e367-e386.

19 Hertenstein E, Feige B, Gmeiner T, et al. Insomnia as a predictor of mental disorders: a systematic review and meta-analysis. Sleep Med Rev 2019; 43: 96-105.

20 Allen RP, Picchietti D, Hening WA, et al. Restless legs syndrome: diagnostic criteria, special considerations, and epidemiology. A report from the restless legs syndrome diagnosis and epidemiology workshop of the National Institute of Health. Sleep Med 2003; 4: 101-119.

21 Allen RP, Montplaisir J, Walters AS, et al. Chapter 95: Restless legs syndrome and periodic limb movements during sleep. In: Kryger M, Roth T, Dement WC, eds. Principles and Practice of Sleep Medicine. 6th Edn. Elsevier, 2017; pp. 923-34.e6.

22 Ohayon MM, O'Hara R, Vitiello MV. Epidemiology of restless legs syndrome: a synthesis of the literature. Sleep Med Rev 2012; 16: 283-295.

23 Ferini-Strambi L, Walters AS, Sica D. The relationship among restless legs syndrome (Willis-Ekbom Disease), hypertension, cardiovascular disease, and cerebrovascular disease. J Neurol 2014; 261: 1051-1068.

24 Trenkwalder C, Allen R, Hogl B, et al. Restless legs syndrome associated with major diseases: a systematic review and new concept. Neurology 2016; 86: 1336-1343.

25 Szentkirályi A, Völzke H, Hoffmann W, et al. Multimorbidity and the risk of restless legs syndrome in 2 prospective cohort studies. Neurology 2014; 82: 2026-2033.

26 Leng Y, Cappuccio FP, Wainwright NW, et al. Sleep duration and risk of fatal and nonfatal stroke: a prospective study and meta-analysis. Neurology 2015; 84: 1072-1079. 
Hermann DM, Bassetti CL. Role of sleep-disordered breathing and sleep-wake disturbances for stroke and stroke recovery. Neurology 2016; 87: 1407-1416. consequences. J Am Coll Cardiol 2017; 69: 841-858.

29 Ma C, Pavlova M, Liu Y, et al. Probable REM sleep behavior disorder and risk of stroke: a prospective study. Neurology 2017; 88: 1849-1855.

30 Duss SB, Seiler A, Schmidt MH, et al. The role of sleep in recovery following ischemic stroke: a review of human and animal data. Neurobiol Sleep Circadian Rhythms 2017; 2: 94-105.

31 Alexiev F, Brill AK, Ott SR, et al. Sleep-disordered breathing and stroke: chicken or egg? J Thorac Dis 2018; 10: Suppl. 34, S4244-S4S52.

32 Yin J, Jin X, Shan Z, et al. Relationship of sleep duration with all-cause mortality and cardiovascular events: a systematic review and dose-response meta-analysis of prospective cohort studies. J Am Heart Assoc 2017; 6: e005947. Kecklund G, Axelsson J. Health consequences of shift work and insufficient sleep. BMJ 2016; 355: i5210.

Koo DL, Nam H, Thomas RJ, et al. Sleep disturbances as a risk factor for stroke. J Stroke 2018; 20: 12-32.

35 Gottlieb E, Landau E, Baxter H, et al. The bidirectional impact of sleep and circadian rhythm dysfunction in human ischaemic stroke: a systematic review. Sleep Med Rev 2019; 45: 54-69.

36 Kernan WN, Ovbiagele B, Black HR, et al. Guidelines for the prevention of stroke in patients with stroke and transient ischemic attack: a guideline for healthcare professionals from the American Heart Association/ American Stroke Association. Stroke 2014; 45: 2160-2236.

37 Leone MA, Brainin M, Boon P, et al. Guidance for the preparation of neurological management guidelines by EFNS scientific task forces - revised recommendations 2012. Eur J Neurol 2013; 20: 410-419.

38 Leone MA, Keindl M, Schapira AH, et al. Practical recommendations for the process of proposing, planning and writing a neurological management guideline by EAN task forces. Eur J Neurol 2015; 22: 1505-1510.

39 Brusselle GG, Gaga M. ERS guidelines, statements and technical standards published in the ERJ in 2014: a year in review. Eur Respir J 2015; 45: 863-866.

40 Shea BJ, Grimshaw JM, Wells GA, et al. Development of AMSTAR: a measurement tool to assess the methodological quality of systematic reviews. BMC Med Res Methodol 2007; 7: 10.

41 Getchius TS, Moses LK, French J, et al. AAN guidelines: a benefit to the neurologist. Neurology 2010; 75: 1126-1127.

42 Partinen M, Palomaki H. Snoring and cerebral infarction. Lancet 1985; 2: 1325-1326.

43 Li M, Li K, Zhang XW, et al. Habitual snoring and risk of stroke: a meta-analysis of prospective studies. Int $J$ Cardiol 2015; 185: 46-49.

44 Loke YK, Brown JW, Kwok CS, et al. Association of obstructive sleep apnea with risk of serious cardiovascular events: a systematic review and meta-analysis. Circ Cardiovasc Qual Outcomes 2012; 5: 720-728.

45 Dong JY, Zhang YH, Qin LQ. Obstructive sleep apnea and cardiovascular risk: meta-analysis of prospective cohort studies. Atherosclerosis 2013; 229: 489-495.

46 Wang X, Ouyang Y, Wang Z, et al. Obstructive sleep apnea and risk of cardiovascular disease and all-cause mortality: a meta-analysis of prospective cohort studies. Int J Cardiol 2013; 169: 207-214.

47 Kendzerska T, Mollayeva T, Gershon AS, et al. Untreated obstructive sleep apnea and the risk for serious long-term adverse outcomes: a systematic review. Sleep Med Rev 2014; 18: 49-59.

48 Li M, Hou WS, Zhang XW, et al. Obstructive sleep apnea and risk of stroke: a meta-analysis of prospective studies. Int J Cardiol 2014; 172: 466-469.

49 Qu H, Guo M, Zhang Y, et al. Obstructive sleep apnea increases the risk of cardiac events after percutaneous coronary intervention: a meta-analysis of prospective cohort studies. Sleep Breath 2018; 22: 33-40.

$50 \mathrm{Xu}$ P. Association of obstructive sleep apnea with incidence of serious cardiovascular events in the elderly: a meta-analysis. Int J Gerontol 2016; 10: 164-169.

51 Wang X, Fan JY, Zhang Y, et al. Association of obstructive sleep apnea with cardiovascular outcomes after percutaneous coronary intervention: A systematic review and meta-analysis. Medicine (Baltimore) 2018 ; 97 : e0621.

52 Xie C, Zhu R, Tian Y, et al. Association of obstructive sleep apnoea with the risk of vascular outcomes and all-cause mortality: a meta-analysis. BMJ Open 2017; 7: e013983.

53 Chang CC, Chuang HC, Lin CL, et al. High incidence of stroke in young women with sleep apnea syndrome. Sleep Med 2014; 15: 410-414.

54 Lamberts M, Nielsen OW, Lip GY, et al. Cardiovascular risk in patients with sleep apnoea with or without continuous positive airway pressure therapy: follow-up of 4.5 million Danish adults. J Intern Med 2014; 276: 659-666.

55 Marshall NS, Wong KK, Cullen SR, et al. Sleep apnea and 20-year follow-up for all-cause mortality, stroke, and cancer incidence and mortality in the Busselton Health Study cohort. J Clin Sleep Med 2014; 10: $355-362$.

56 Molnar MZ, Mucsi I, Novak M, et al. Association of incident obstructive sleep apnoea with outcomes in a large cohort of US veterans. Thorax 2015; 70: 888-895.

57 Campos-Rodriguez F, Martinez-Garcia MA, Reyes-Nunez N, et al. Role of sleep apnea and continuous positive airway pressure therapy in the incidence of stroke or coronary heart disease in women. Am J Respir Crit Care Med 2014; 189: 1544-1550.

58 Loo G, Tan AY, Koo CY, et al. Prognostic implication of obstructive sleep apnea diagnosed by post-discharge sleep study in patients presenting with acute coronary syndrome. Sleep Med 2014; 15: 631-636.

59 Lee $\mathrm{CH}$, Sethi R, Li R, et al. Obstructive sleep apnea and cardiovascular events after percutaneous coronary intervention. Circulation 2016; 133: 2008-2017.

60 Kendzerska T, Gershon AS, Hawker G, et al. Obstructive sleep apnea and risk of cardiovascular events and all-cause mortality: a decade-long historical cohort study. PLoS Med 2014; 11: e1001599.

61 Lee JE, Lee CH, Lee SJ, et al. Mortality of patients with obstructive sleep apnea in Korea. J Clin Sleep Med 2013; 9: $997-1002$.

62 Lipford MC, Flemming KD, Calvin AD, et al. Associations between cardioembolic stroke and obstructive sleep apnea. Sleep 2015; 38: 1699-1705.

63 Yaranov DM, Smyrlis A, Usatii N, et al. Effect of obstructive sleep apnea on frequency of stroke in patients with atrial fibrillation. Am J Cardiol 2015; 115: 461-465. 
65 Tuohy CV, Montez-Rath ME, Turakhia M, et al. Sleep disordered breathing and cardiovascular risk in older patients initiating dialysis in the United States: a retrospective observational study using medicare data. BMC Nephrol 2016; 17: 16.

66 Stone KL, Blackwell TL, Ancoli-Israel S, et al. Sleep disordered breathing and risk of stroke in older community-dwelling men. Sleep 2016; 39: 531-540.

67 Marin JM, Carrizo SJ, Vicente E, et al. Long-term cardiovascular outcomes in men with obstructive sleep apnoea-hypopnoea with or without treatment with continuous positive airway pressure: an observational study. Lancet 2005; 365: 1046-1053.

68 McEvoy RD, Antic NA, Heeley E, et al. CPAP for prevention of cardiovascular events in obstructive sleep apnea. N Engl J Med 2016; 375: 919-931.

69 Abuzaid AS, Al Ashry HS, Elbadawi A, et al. Meta-analysis of cardiovascular outcomes with continuous positive airway pressure therapy in patients with obstructive sleep apnea. Am J Cardiol 2017; 120: 693-699.

70 Halle TR, Oh MS, Collop NA, et al. Surgical treatment of OSA on cardiovascular outcomes: a systematic review. Chest 2017; 152: 1214-1229.

71 Kim Y, Koo YS, Lee HY, et al. Can continuous positive airway pressure reduce the risk of stroke in obstructive sleep apnea patients? A systematic review and meta-analysis. PLoS One 2016; 11: e0146317.

72 Khan SU, Duran CA, Rahman $\mathrm{H}$, et al. A meta-analysis of continuous positive airway pressure therapy in prevention of cardiovascular events in patients with obstructive sleep apnoea. Eur Heart J 2018; 39: 2291-2297.

73 Catalan-Serra P, Campos-Rodriguez F, Reyes-Nunez N, et al. Increased incidence of stroke, but not coronary heart disease, in elderly patients with sleep apnea. Stroke 2019; 50: 491-494.

$74 \mathrm{Wu} \mathrm{X}, \mathrm{Lv} \mathrm{S}, \mathrm{Yu} \mathrm{X}$, et al. Treatment of OSA reduces the risk of repeat revascularization after percutaneous coronary intervention. Chest 2015; 147: 708-718.

75 Chen SY, Cherng YG, Lee FP, et al. Risk of cerebrovascular diseases after uvulopalatopharyngoplasty in patients with obstructive sleep apnea: a nationwide cohort study. Medicine (Baltimore) 2015; 94: el791.

76 Partinen M, Guilleminault C. Daytime sleepiness and vascular morbidity at seven-year follow-up in obstructive sleep apnea patients. Chest 1990; 97: 27-32.

77 Gottlieb DJ, Punjabi NM, Mehra R, et al. CPAP versus oxygen in obstructive sleep apnea. N Engl J Med 2014 370: 2276-2285.

78 Parra O, Sanchez-Armengol A, Capote F, et al. Efficacy of continuous positive airway pressure treatment on 5-year survival in patients with ischaemic stroke and obstructive sleep apnea: a randomized controlled trial. J Sleep Res 2015; 24: 47-53.

79 Peker Y, Glantz H, Eulenburg C, et al. Effect of positive airway pressure on cardiovascular outcomes in coronary artery disease patients with nonsleepy obstructive sleep apnea. The RICCADSA randomized controlled trial. Am J Respir Crit Care Med 2016; 194: 613-620.

80 Huang Z, Liu Z, Luo Q, et al. Long-term effects of continuous positive airway pressure on blood pressure and prognosis in hypertensive patients with coronary heart disease and obstructive sleep apnea: a randomized controlled trial. Am J Hypertens 2015; 28: 300-306.

81 Parra O, Sanchez-Armengol A, Bonnin M, et al. Early treatment of obstructive apnoea and stroke outcome: a randomised controlled trial. Eur Respir J 2011; 37: 1128-1136.

82 Martinez-Garcia MA, Campos-Rodriguez F, Catalan-Serra P, et al. Cardiovascular mortality in obstructive sleep apnea in the elderly: role of long-term continuous positive airway pressure treatment: a prospective observational study. Am J Respir Crit Care Med 2012; 186: 909-916.

83 Buchner NJ, Sanner BM, Borgel J, et al. Continuous positive airway pressure treatment of mild to moderate obstructive sleep apnea reduces cardiovascular risk. Am J Respir Crit Care Med 2007; 176: 1274-1280.

84 Doherty LS, Kiely JL, Swan V, et al. Long-term effects of nasal continuous positive airway pressure therapy on cardiovascular outcomes in sleep apnea syndrome. Chest 2005; 127: 2076-2084.

85 Kripke DF, Garfinkel L, Wingard DL, et al. Mortality associated with sleep duration and insomnia. Arch Gen Psychiatry 2002; 59: 131-136.

86 Vgontzas AN, Liao D, Bixler EO, et al. Insomnia with objective short sleep duration is associated with a high risk for hypertension. Sleep 2009; 32: 491-497.

87 He Q, Zhang P, Li G, et al. The association between insomnia symptoms and risk of cardio-cerebral vascular events: a meta-analysis of prospective cohort studies. Eur J Prev Cardiol 2017; 24: 1071-1082.

88 Kwok CS, Kontopantelis E, Kuligowski G, et al. Self-reported sleep duration and quality and cardiovascular disease and mortality: a dose-response meta-analysis. J Am Heart Assoc 2018; 7: e008552.

89 Wu MP, Lin HJ, Weng SF, et al. Insomnia subtypes and the subsequent risks of stroke: report from a nationally representative cohort. Stroke 2014; 45: 1349-1354.

90 Hsu CY, Chen YT, Chen $\mathrm{MH}$, et al. The association between insomnia and increased future cardiovascular events: a nationwide population-based study. Psychosom Med 2015; 77: 743-751.

91 Helbig AK, Stockl D, Heier M, et al. Symptoms of insomnia and sleep duration and their association with incident strokes: findings from the population-based MONICA/KORA Augsburg cohort study. PLoS One 2015; 10: e0134480.

92 Westerlund A, Bellocco R, Sundstrom J, et al. Sleep characteristics and cardiovascular events in a large Swedish cohort. Eur J Epidemiol 2013; 28: 463-473.

93 Barker MJ, Greenwood KM, Jackson M, et al. Persistence of cognitive effects after withdrawal from long-term benzodiazepine use: a meta-analysis. Arch Clin Neuropsychol 2004; 19: 437-454.

94 Woodward ND, Purdon SE, Meltzer HY, et al. A meta-analysis of neuropsychological change to clozapine, olanzapine, quetiapine, and risperidone in schizophrenia. Int J Neuropsychopharmacol 2005; 8: 457-472.

95 Lucchetta RC, da Mata BPM, Mastroianni PC. Association between development of dementia and use of benzodiazepines: a systematic review and meta-analysis. Pharmacotherapy 2018; 38: 1010-1020.

96 Parsaik AK, Mascarenhas SS, Hashmi A, et al. Role of botulinum toxin in depression. J Psychiatr Pract 2016; 22: 99-110. 
Huang WS, Muo CH, Chang SN, et al. Benzodiazepine use and risk of stroke: a retrospective population-based cohort study. Psychiatry Clin Neurosci 2014; 68: 255-262.

98 Zhu J, Jiang J, Zhang Y, et al. Non-benzodiazepine hypnotic drug is correlated with decreased risk of ischemic stroke. Int J Clin Exp Med 2016; 9: 23777-23780.

99 Taipale H, Koponen M, Tanskanen A, et al. Use of benzodiazepines and related drugs is associated with a risk of stroke among persons with Alzheimer's disease. Int Clin Psychopharmacol 2017; 32: 135-141.

100 Gottlieb DJ, Somers VK, Punjabi NM, et al. Restless legs syndrome and cardiovascular disease: a research roadmap. Sleep Med 2017; 31: 10-17.

101 Kendzerska T, Kamra M, Murray BJ, et al. Incident cardiovascular events and death in individuals with restless legs syndrome or periodic limb movements in sleep: a systematic review. Sleep 2017; 40: zsx013.

102 Katsanos AH, Kosmidou M, Konitsiotis S, et al. Restless legs syndrome and cerebrovascular/cardiovascular events: systematic review and meta-analysis. Acta Neurol Scand 2018; 137: 142-148.

103 Lin TC, Zeng BY, Chen YW, et al. Cerebrovascular accident risk in a population with periodic limb movements of sleep: a preliminary meta-analysis. Cerebrovasc Dis 2018; 46: 1-9.

104 Winkelman JW, Blackwell T, Stone K, et al. Associations of incident cardiovascular events with restless legs syndrome and periodic leg movements of sleep in older men, for the outcomes of sleep disorders in older men study (MrOS Sleep Study). Sleep 2017; 40: 10.1093/sleep/zsx023.

105 Cholley-Roulleau M, Chenini S, Beziat S, et al. Restless legs syndrome and cardiovascular diseases: a case-control study. PLoS One 2017; 12: e0176552.

106 Bassetti C, Aldrich MS, Chervin RD, et al. Sleep apnea in patients with transient ischemic attack and stroke: a prospective study of 59 patients. Neurology 1996; 47: 1167-1173.

107 Dyken ME, Somers VK, Yamada T, et al. Investigating the relationship between stroke and obstructive sleep apnea. Stroke 1996; 27: 401-407.

108 Johnson KG, Johnson DC. Frequency of sleep apnea in stroke and TIA patients: a meta-analysis. J Clin Sleep Med 2010; 6: 131-137.

109 Seiler A, Camilo M, Korostovtseva L, et al. Prevalence of sleep-disordered breathing after stroke and TIA: a meta-analysis. Neurology 2019; 92: e648-ee54.

110 Boulos MI, Elias S, Wan A, et al. Unattended hospital and home sleep apnea testing following cerebrovascular events. J Stroke Cerebrovasc Dis 2017; 26: 143-149.

111 Festic N, Alejos D, Bansal V, et al. Sleep apnea in patients hospitalized with acute ischemic stroke: underrecognition and associated clinical outcomes. J Clin Sleep Med 2018; 14: 75-80.

112 Huhtakangas JK, Huhtakangas J, Bloigu R, et al. Prevalence of sleep apnea at the acute phase of ischemic stroke with or without thrombolysis. Sleep Med 2017; 40: 40-46.

113 Kumar R, Suri JC, Manocha R. Study of association of severity of sleep disordered breathing and functional outcome in stroke patients. Sleep Med 2017; 34: 50-56.

114 Menon D, Sukumaran S, Varma R, et al. Impact of obstructive sleep apnea on neurological recovery after ischemic stroke: a prospective study. Acta Neurol Scand 2017; 136: 419-426.

115 Scherbakov N, Sandek A, Ebner N, et al. Sleep-disordered breathing in acute ischemic stroke: a mechanistic link to peripheral endothelial dysfunction. J Am Heart Assoc 2017; 6: e006010.

116 Birkbak J, Clark AJ, Rod NH. The effect of sleep disordered breathing on the outcome of stroke and transient ischemic attack: a systematic review. J Clin Sleep Med 2014; 10: 103-108.

117 Xie W, Zheng F, Song X. Obstructive sleep apnea and serious adverse outcomes in patients with cardiovascular or cerebrovascular disease: a PRISMA-compliant systematic review and meta-analysis. Medicine (Baltimore) 2014; 93 : e336.

118 Kim TJ, Ko SB, Jeong HG, et al. Nocturnal desaturation is associated with neurological deterioration following ischemic stroke: a retrospective observational study. J Clin Sleep Med 2017; 13: 1273-1279.

119 Ponsaing LB, Iversen HK, Jennum P. Polysomnographic indicators of mortality in stroke patients. Sleep Breath 2017; 21: 235-242.

120 Tsivgoulis G, Alexandrov AV, Katsanos AH, et al. Noninvasive ventilatory correction in patients with acute ischemic stroke: a systematic review and meta-analysis. Stroke 2017; 48: 2285-2288.

121 Brill AK, Horvath T, Seiler A, et al. CPAP as treatment of sleep apnea after stroke: a meta-analysis of randomized trials. Neurology 2018; 90: e1222-e1e30.

122 Bravata DM, Sico J, Vaz Fragoso CA, et al. Diagnosing and treating sleep apnea in patients with acute cerebrovascular disease. J Am Heart Assoc 2018; 7: e008841.

123 Gupta A, Shukla G, Afsar M, et al. Role of positive airway pressure therapy for obstructive sleep apnea in patients with stroke: a randomized controlled trial. J Clin Sleep Med 2018; 14: 511-521.

124 Svatikova A, Chervin RD, Wing JJ, et al. Positional therapy in ischemic stroke patients with obstructive sleep apnea. Sleep Med 2011; 12: 262-266.

125 Frohnhofen H, Höltmann B, Orth G, et al. Nächtliche Sauerstofftherapie und kognitive Funktion bei bewußtseinsklaren, älteren Patienten mit Hirninfarkt und obstruktiver Schlafapnoe-Eine kontrollierte Studie [Nocturnal oxygen therapy and cognitive function in conscious elderly patients with ischemic stroke and obstructive sleep apnea (OSA) - a controlled study]. Somnologie - Schlafforschung und Schlafmedizin 1998; 2: $172-183$.

126 Brunner H. Success and failure of mirtazapine as alternative treatment in elderly stroke patients with sleep apnea-a preliminary open trial. Sleep Breath 2008; 12: 281-285.

127 Haba-Rubio J, Andries D, Rey V, et al. Effect of transnasal insufflation on sleep disordered breathing in acute stroke: a preliminary study. Sleep Breath 2012; 16: 759-764.

128 Brill AK, Rosti R, Hefti JP, et al. Adaptive servo-ventilation as treatment of persistent central sleep apnea in post-acute ischemic stroke patients. Sleep Med 2014; 15: 1309-1313.

129 Wheeler NC, Wing JJ, O’Brien LM, et al. Expiratory positive airway pressure for sleep apnea after stroke: a randomized, crossover trial. J Clin Sleep Med 2016; 12: 1233-1238.

130 Ye D, Chen C, Song D, et al. Oropharyngeal muscle exercise therapy improves signs and symptoms of post-stroke moderate obstructive sleep apnea syndrome. Front Neurol 2018; 9: 912.

131 Baglioni C, Nissen C, Schweinoch A, et al. Polysomnographic characteristics of sleep in stroke: a systematic review and meta-analysis. PLoS One 2016; 11: e0148496. 
132 Tang WK, Grace Lau C, Mok V, et al. Insomnia and health-related quality of life in stroke. Top Stroke Rehabil 2015; 22: 201-207.

133 Tang WK, Lu JY, Liang H, et al. Is insomnia associated with suicidality in stroke? Arch Phys Med Rehabil 2011; 92: 2025-2027.

134 Glozier N, Moullaali TJ, Sivertsen B, et al. The course and impact of poststroke insomnia in stroke survivors aged 18 to 65 years: results from the Psychosocial Outcomes In StrokE (POISE) study. Cerebrovasc Dis Extra 2017; 7: 9-20.

135 Leppavuori A, Pohjasvaara T, Vataja R, et al. Insomnia in ischemic stroke patients. Cerebrovasc Dis 2002; 14: 90-97.

136 Palomaki H, Berg A, Meririnne E, et al. Complaints of poststroke insomnia and its treatment with mianserin. Cerebrovasc Dis 2003; 15: 56-62.

137 Da Rocha PC, Barroso MT, Dantas AA, et al. Predictive factors of subjective sleep quality and insomnia complaint in patients with stroke: implications for clinical practice. An Acad Bras Cienc 2013; 85: 1197-1206.

138 Kim KT, Moon HJ, Yang JG, et al. The prevalence and clinical significance of sleep disorders in acute ischemic stroke patients-a questionnaire study. Sleep Breath 2017; 21: 759-765.

139 Joa KL, Kim WH, Choi HY, et al. The effect of sleep disturbances on the functional recovery of rehabilitation inpatients following mild and moderate stroke. Am J Phys Med Rehabil 2017; 96: 734-740.

140 Li LJ, Yang Y, Guan BY, et al. Insomnia is associated with increased mortality in patients with first-ever stroke: a 6-year follow-up in a Chinese cohort study. Stroke Vasc Neurol 2018; 3: 197-202.

141 Lodder J, van Raak L, Hilton A, et al. Diazepam to improve acute stroke outcome: results of the early GABA-Ergic activation study in stroke trial. A randomized double-blind placebo-controlled trial. Cerebrovasc Dis 2006; 21: 120-127.

142 Clarkson AN, Huang BS, Macisaac SE, et al. Reducing excessive GABA-mediated tonic inhibition promotes functional recovery after stroke. Nature 2010; 468: 305-309.

143 Wang YC, Dzyubenko E, Sanchez-Mendoza EH, et al. Postacute delivery of GABAA alpha5 antagonist promotes postischemic neurological recovery and peri-infarct brain remodeling. Stroke 2018; 49: 2495-2503.

144 Lazar RM, Fitzsimmons BF, Marshall RS, et al. Midazolam challenge reinduces neurological deficits after transient ischemic attack. Stroke 2003; 34: 794-796.

145 Lazar RM, Berman MF, Festa JR, et al. GABAergic but not anti-cholinergic agents re-induce clinical deficits after stroke. J Neurol Sci 2010; 292: 72-76.

146 Liu J, Wang LN, Ma X, et al. Gamma aminobutyric acid (GABA) receptor agonists for acute stroke. Cochrane Database Syst Rev 2016; 10: CD009622.

147 Kim CR, Chun MH, Han EY. Effects of hypnotics on sleep patterns and functional recovery of patients with subacute stroke. Am J Phys Med Rehabil 2010; 89: 315-322.

148 Schlesinger I, Erikh I, Nassar M, et al. Restless legs syndrome in stroke patients. Sleep Med 2015; 16: 1006-1010.

149 Lee SJ, Kim JS, Song IU, et al. Poststroke restless legs syndrome and lesion location: anatomical considerations. Mov Disord 2009; 24: 77-84.

150 Medeiros CA, de Bruin PF, Paiva TR, et al. Clinical outcome after acute ischaemic stroke: the influence of restless legs syndrome. Eur J Neurol 2011; 18: 144-149.

151 Gupta A, Shukla G, Mohammed A, et al. Restless legs syndrome, a predictor of subcortical stroke: a prospective study in 346 stroke patients. Sleep Med 2017; 29: 61-67.

152 Boulos MI, Wan A, Black SE, et al. Restless legs syndrome after high-risk TIA and minor stroke: association with reduced quality of life. Sleep Med 2017; 37: 135-140.

153 Boulos MI, Murray BJ, Muir RT, et al. Periodic limb movements and white matter hyperintensities in first-ever minor stroke or high-risk transient ischemic attack. Sleep 2017; 40: zsw080.

154 Manconi M, Fanfulla F, Ferri R, et al. Periodic limb movements during sleep in stroke/TIA: prevalence, course, and cardiovascular burden. Neurology 2018; 90: e1663-ele72.

155 Shiina T, Suzuki K, Okamura M, et al. Restless legs syndrome and its variants in acute ischemic stroke. Acta Neurol Scand 2019; 139: 260-268.

156 Woo HG, Lee D, Hwang KJ, et al. Post-stroke restless leg syndrome and periodic limb movements in sleep. Acta Neurol Scand 2017; 135: 204-210.

157 Tuo HZ, Tian ZL, Cui YN, et al. Restless legs syndrome secondary to pontine infarction: Clinical analysis of five cases. Chronic Dis Transl Med 2017; 3: 186-190.

158 Bassetti CLA. Sleep and stroke: a bidirectional relationship with clinical implications. Sleep Med Rev 2019; 45: $127-128$. 